\title{
A global invariant for three dimensional CR-manifolds
}

\author{
D.M. Burns, Jr. ${ }^{1}$ and C.L. Epstein ${ }^{2, \star}$ \\ ${ }^{1}$ Department of Mathematics, University of Michigan, Ann Arbor, MI 48109, USA \\ ${ }^{2}$ Department of Mathematics, The University of Pennsylvania, Philadelphia, PA 19104, USA
}

\section{\$1. Introduction}

In this note we define a global, $\mathbf{R}$-valued invariant of a compact, strictly pseudoconvex 3-dimensional CR-manifold $M$ whose holomorphic tangent bundle is trivial. The invariant arises as the evaluation of a deRham cohomology class on the fundamental class of the manifold. To construct the relevant form, we start with the $\mathrm{CR}$ structure bundle $Y$ over $M$ (see [Ch-Mo], whose notation we follow). The form is a secondary characteristic form of this structure. By fixing a contact form and coframe, i.e., a section of $Y$, we obtain a form on $M$. Surprisingly, this form is well-defined up to an exact term, and thus its cohomology class is well-defined in $H^{3}(M, \mathbf{R})$.

Our motivation for studying this invariant was its analogy with the $\mathbf{R} / \mathbf{Z}$ secondary characteristic number associated by Chern and Simons to the conformal class of a Riemannian 3-manifold $N$, which provides an obstruction to the conformal immersion of $N$ in $\mathbf{R}^{4}$. Though several formal analogies to the conformal case are valid for our invariant, this one does not hold up: specifically, in $\$ 4$ below, we calculate examples which show that the CR invariant can take on any positive real value for hypersurfaces embedded in $\mathbf{C}^{2}$. It is also clear that the invariant is neither a homotopy nor concordance invariant, but depends in an elusive way on the CR structure. Our inspiration came from the seminal papers of Chern and Moser and Chern and Simons. The idea of looking at secondary characteristic forms of higher order geometric structures in general appears in [Ko-Oc], though with a different intention.

In $\S 2$ we will quickly review the definition of a CR structure, the construction of $Y$ and its reduction to a pseudo-hermitian structure $a$ là Webster [We].

In $\S 3$ we define the invariant and prove that it is, in fact, $\mathbf{R}$-valued, and not $\mathbf{R} / \mathbf{Z}$-valued as in the Riemannian case. We also prove that if the invariant is stationary as a function of the CR structure, then $M$ is locally CR equivalent to the standard three sphere in $\mathbf{C}^{2}$, paralleling a result of Chern and Simons.

As noted already, $\S 4$ is devoted to the calculation of several examples.

\footnotetext{
* Research partially supported by the National Science Foundation, grants DMS 84-01978 (DB) and DMS 86-00338 (CE)
} 
The bulk of the work on invariants of CR structures has been directed towards the construction of local invariants, though Donelly and S.S.-T. Yau have considered global invariants. Donelly's invariant is analogous to the eta invariant; it is a homotopy invariant, and vanishes $\bmod \mathbf{Q}$ for $\mathrm{CR}$ manifolds that locally bound complex varieties, cf. [Do]. Yau related the dimension of Kohn-Rossi cohomology groups of boundaries of complex varieties to the singularites of the variety, cf. [Y].

As will be clear from the construction, many such invariants can be defined for non-degenerate CR-structures on manifolds of any odd dimension, though we have not checked yet whether, e.g., the classes so defined could be R-valued. There is a classifying space approach to these constructions which indicates that these classes are markedly different from their Riemannian counterparts. This will be discussed in a later publication.

For a structure induced from an immersion in a complex manifold, the differential form defining the cohomology class depends on four derivatives of the immersion. This is of interest in light of the classical result of E. Cartan that a 3-dimensional $C R$ manifold has no local invariants of derivative order less than six, cf. [Ch-Mo].

Acknowledgements. The second named author would like to thank J.J. Kohn, Dave Barrett, John D'Angelo and Robin Graham for introducing him to CR geometry, and generously sharing their various personal outlooks on the subject with him. We would also like to thank Robert Bryant and John Scherk for pointing out corrections to the first draft of this paper.

\section{§ 2. Background}

A CR structure on a $2 n+1$-dimensional manifold $M$ is defined by choosing an $n$-dimensional subbundle $T^{1,0} M$ of the complexified tangent bundle of $M$. This is the bundle of holomorphic tangent spaces, and must have the following properties:

1) $T^{1,0} M \cap \overline{T^{1,0} M}=\{0\}$

2) If $X, Y$ are two sections of $T^{1,0} M$, then so is $[X, Y]$.

If $\theta$ is a real one-form annihilating $T^{1,0} M \oplus T^{1,0} \bar{M}$, then the structure is strictly pseudoconvex if $d \theta$ defines a positive definite pairing of $T^{1,0} M$ with $\overline{T^{1,0} M}$. The form $\theta$ is called a contact form; $d \theta$ is the Levi form. If $\left\{X_{1}, \ldots, X_{n}\right\}$ is a local frame field for $T^{1,0} M$ and $\left\{\theta_{1}, \ldots, \theta_{n}\right\}$ is a dual coframe field, then property 2) implies that:

$$
d \theta=i g_{\alpha \bar{\beta}} \theta^{\alpha} \wedge \theta^{\bar{\beta}}+\theta \wedge \phi
$$

where $\theta^{\tilde{\beta}}=\overline{\theta^{\beta}}$ and $\phi$ is a real one form; $\boldsymbol{g}_{\alpha \bar{\beta}}=\overline{\boldsymbol{g}_{\beta \bar{\alpha}}}$. If the structure is strictly pseudoconvex, then $\mathrm{g}_{\alpha \bar{\beta}}$ is positive definite. In [Ch-Mo] the equivalence problem for non-degenerate CR structures is solved. We will quickly review the results for the special case of a 3-dimensional $C R$ manifold, already treated by $E$. Cartan [C].

In this case (2.1) reduces to:

$$
d \theta=i g_{1 \overline{1}} \theta^{1} \wedge \theta^{\tilde{1}}+\theta \wedge \phi .
$$




\section{The CR structure bundle}

As there is no canonical choice of contact form one is led to introduce the trivial line bundle $E \rightarrow M$ defined by

$$
M \times \mathbf{R} \ni(p, t) \rightarrow\left(e^{t} \theta_{p}, p\right) \in E \subset T^{*} M .
$$

Let $\omega$ denote the tautological one form on $E$. The CR structure bundle $Y$ over $E$ is then defined as the set of coframes $\left(\omega, \omega^{1}, \omega^{\overline{1}}, \phi\right)$ such that:

$$
d \omega=i g_{1 \check{1}} \omega^{1} \wedge \omega^{\overline{1}}+\omega \wedge \phi
$$

The "matrix" $g_{1 \overline{1}}$ is fixed, we will henceforth take it to be 1 . The forms $\omega^{1}$, $\omega^{\overline{1}}$ are in the ideal generated by $\theta^{1}$ and $\theta^{\overline{1}}$ pulled back from $T^{*} M$ to $T^{*} E$. The structure group of $Y$ is the group of matrices:

$$
\left(\begin{array}{cccc}
1 & 0 & 0 & 0 \\
v^{1} & e^{i \alpha} & 0 & 0 \\
v^{\overline{1}} & 0 & e^{-i \alpha} & 0 \\
s & i e^{i \alpha} v^{\overline{1}} & -i e^{-i \alpha} v^{1} & 1
\end{array}\right)
$$

where $s$ and $\alpha$ are real. In $Y$ one can introduce connection forms $\left\{\phi_{1}^{1}, \phi^{1}\right.$, $\psi$ ) such that:

and

$$
d \omega^{1}=\omega^{1} \wedge \phi_{1}^{1}+\omega \wedge \phi^{1}
$$

$$
d \phi=i \omega_{\overline{1}} \wedge \phi^{\overline{1}}+i \phi_{\overline{1}} \wedge \omega^{\overline{1}}+\omega \wedge \psi
$$

These forms are normalized by

$$
\phi-\phi_{1}^{1}-\phi_{\overline{1}}^{\overline{1}}=0
$$

To determine completely the connection forms one introduces various curvature forms:

and

$$
\begin{aligned}
\Phi_{1}^{1} & =d \phi_{1}^{1}-i \omega_{1} \wedge \phi^{1}+2 i \phi_{1} \wedge \omega^{1}+1 / 2 \psi \wedge \omega \\
& =S_{11 \overline{1}}^{1} \omega^{1} \wedge \omega^{\overline{1}}+V_{11}^{1} \omega^{1} \wedge \omega-V_{1 \overline{1}}^{1} \omega^{\overline{1}} \wedge \omega
\end{aligned}
$$

$$
\begin{aligned}
\Phi^{1} & =d \phi^{1}-\phi \wedge \phi^{1}-\phi^{1} \wedge \phi_{1}^{1}+1 / 2 \psi \wedge \omega^{1} \\
& =V_{1 \overline{1}}^{1} \omega^{1} \wedge \omega^{\overline{1}}+P_{1}^{1} \omega^{1} \wedge \omega+Q_{\overline{1}}^{1} \omega^{\overline{1}} \wedge \omega
\end{aligned}
$$

The conditions $S_{11 \overline{1} 1}=V_{1 \overline{1}}=P_{1 \overline{1}}=0$ uniquely determine the connection forms. With these forms one can define an $\mathfrak{s} \mathfrak{u}(2,1)$-valued Cartan connection form on $Y$ via:

$$
\pi=\left(\begin{array}{ccc}
-1 / 3\left(\phi_{1}^{1}+\phi\right) & \omega^{1} & 2 \omega \\
-i \phi_{1} & 1 / 3\left(2 \phi_{1}^{1}-\phi\right) & 2 i \omega_{1} \\
-1 / 4 \psi & 1 / 2 \phi^{1} & 1 / 3\left(\phi+\frac{1}{1} \phi\right)
\end{array}\right)
$$


The curvature of this connection is then:

$$
\Pi=d \pi+\pi \wedge \pi=\left(\begin{array}{ccc}
-1 / 3 \Phi_{1}^{1} & 0 & 0 \\
-\mathrm{i} \Phi_{1} & 2 / 3 \Phi_{1}^{1} & 0 \\
-1 / 4 \Psi & 1 / 2 \Phi^{1} & -1 / 3 \Phi_{\overline{1}}^{\overline{1}}
\end{array}\right)
$$

In three dimensions this reduces to:

$$
\Pi=\left(\begin{array}{ccc}
0 & 0 & 0 \\
-i \Phi_{1} & 0 & 0 \\
-1 / 4 \Psi & 1 / 2 \Phi^{1} & 0
\end{array}\right)
$$

\section{Pseudo-Hermitian structures}

In [We] a reduction of the structure group of $Y$ to $U(1)$ is effected (for threedimensional $\mathrm{CR}$ manifolds) by fixing a contact form $\theta$ and requiring $\phi \equiv 0$ in (2.1'). We consider coframes for $T^{1,0} M,\left\{\theta^{1}, \theta^{\overline{1}}\right\}$ so that

$$
d \theta=i \theta^{1} \wedge \theta^{\overline{1}}
$$

Webster defines connection and torsion forms via the equations:

normalized by:

$$
d \theta^{1}=\theta^{1} \wedge \theta_{1}^{1}+\theta \wedge \tau^{1}
$$

and

$$
\left.\begin{array}{l}
\theta_{1}^{1}+\theta_{\overline{1}}^{\overline{1}}=0 \\
\tau^{1} \wedge \theta^{\overline{1}}=0
\end{array}\right\}
$$

We will call the bundle of such coframes $X \rightarrow M$. The curvature of this connection is:

$$
\Omega_{1}^{1}=d \theta_{1}^{1}=R \theta^{1} \wedge \theta^{\overline{1}}+W \theta^{1} \wedge \theta-\bar{W} \theta^{\overline{1}} \wedge \theta .
$$

The choice of $\theta$ defines a section of the bundle $E \rightarrow M$. The bundle $Y$ over this section can be regarded as a vector bundle over $X$. Webster included $X$ into $Y$ by setting:

$$
\left.\begin{array}{rl}
\phi_{1}^{1} & =\theta_{1}^{1}+i / 4 R \theta \\
\phi^{1} & =\tau^{1}+i / 4 R \theta^{1}+E \theta \\
\psi & =G \theta+i\left(\bar{E} \theta^{1}-E \theta^{\overline{1}}\right) .
\end{array}\right\}
$$

The coefficient $E$ is determined from $\Phi_{1}^{1}=0$. The equation for $\psi$ is a correction of Eq. (3.3) in [We] where it is incorrectly asserted that $\psi$ can be made to vanish. The inclusion of $X$ into $Y$ above is a homotopy equivalence. $Y$ and its connection are biholomorphically invariant, whereas the pseudo-hermitian structure is not. Our strategy is to define a form on $Y$ and then via (2.i3) pull it down to $X$ and ultimately to $M$. 


\section{§3. The global invariant}

Since the curvature $\Pi$ of $\pi$ is trace-free, the second Chern form of $\Pi$ is given by

$$
C_{2}(\Pi)=\frac{1}{8 \pi^{2}} \operatorname{tr}(\Pi \wedge \Pi) \text {. }
$$

The usual arguments show that $C_{2}(I)$ is a closed, basic form (with respect to $E$ ). Moreover, one easily shows:

Lemma 3.1. For $\Pi$ and $\pi$ given by (2.7) and $\left(2.8^{\prime}\right)$ we have

$$
C_{2}(\Pi)=d T C_{2}(\pi)
$$

where

$$
T C_{2}(\pi)=\frac{1}{8 \pi^{2}}[\operatorname{tr}(\pi \wedge \Pi)+1 / 3 \operatorname{tr}(\pi \wedge \pi \wedge \pi)] .
$$

Moreover, $C_{2}(\Pi) \equiv 0$.

The arguments are standard; for the convenience of the reader we include a proof.

Proof of Lemma 3.1. The last assertion, that the form $C_{2}(I)$ vanishes identically follows easily from the lower triangular form of $\Pi$ (cf. (2.8') above; note that, as $\operatorname{dim} E=4$, this is not the tautology it usually is). To prove (3.1) we use the facts

1) $d \Pi=\pi \wedge \pi-\pi \wedge \Pi$

2) $\operatorname{tr}(\pi \wedge \Pi \wedge \pi)=\operatorname{tr}(\pi \wedge \pi \wedge \Pi)=0$

3) $\operatorname{tr}(\psi \wedge \phi)=(-1)^{k l} \operatorname{tr}(\phi \wedge \psi)$, if $\psi$ is a matrix of $k$-forms and $\phi$ is a matrix of $l$-forms.

Assertion 1) is the Bianchi identity (differentiate (2.8)); assertion 2) follows by differentiating $\operatorname{tr}(\Pi \wedge \pi)=0$, which in turn follows from $\left(2.8^{\prime}\right)$ and the fact that $\omega \wedge \Psi=\omega^{\overline{1}} \wedge \Phi^{1}=0$.

To prove the lemma, we calculate that:

$$
d T C_{2}(\pi)=\frac{1}{8 \pi^{2}}[\operatorname{tr}(d \pi \wedge \Pi)-\operatorname{tr}(\pi \wedge d \Pi)+\operatorname{tr}(d \pi \wedge \pi \wedge \pi)]
$$

which, in virtue of 1) and 2),

$$
=\frac{1}{8 \pi^{2}}[\operatorname{tr}(d \pi \wedge \Pi)-\operatorname{tr}(\pi \wedge \pi \wedge \pi \wedge \pi)]
$$

Hence, from (2.8), and 2) and 3) above it follows that:

$$
d T C_{2}(\pi)=\frac{1}{8 \pi^{2}} \operatorname{tr}(\Pi \wedge \Pi) .
$$

As a consequence of the lemma we see that $T C_{2}(\pi)$ defines a closed, biholomorphically invariant three form on $Y$. If $Y$ is homotopically equivalent to $M$ $\times S^{1}$, one can hope to pull this closed form down to $M$. A priori the cohomology 
class of $T C_{2}(\pi)$ may be affected by the circle factor. This turns out not to be the case. Using (2.7) one easily shows that along a section of $Y$ where $\phi$ restricts to zero we have:

$$
T C_{2}(\pi)=\frac{1}{8 \pi^{2}}\left[\left(\omega^{1} \wedge \phi^{\overline{1}}+\omega^{\overline{1}} \wedge \phi^{1}\right) \wedge \phi_{1}^{1}-2 \omega \wedge \phi^{1} \wedge \phi^{\overline{1}}-1 / 2 d(\omega \wedge \psi)\right] .
$$

We have used the relation $d \psi=2 i \phi^{1} \wedge \phi^{\overline{1}} \bmod \omega$, which is a consequence of (2.8) and the trace conditions on $\Pi$, and the fact, once again, that $\operatorname{tr}(\Pi \wedge \pi)=0$.

To pull this form down to $X$, the pseudo-hermitian structure bundle, we need to determine the coefficient $E$ appearing in (2.13); the coefficient $G$ turns out to be unimportant here. Solving $\Phi_{1}^{1}=0$ with $\phi_{1}^{1}, \phi^{1}$ and $\psi$ given by (2.13), we obtain:

$$
E=\frac{R_{\overline{1}}}{6}+\frac{2 i \bar{W}}{3}
$$

where

$$
d R=R_{1} \theta^{1}+R_{\overline{1}} \theta^{\overline{1}}+R_{0} \theta
$$

for $\left\{\theta, \theta^{1}, \theta^{\overline{1}}\right\}$ an element of $X$. With these formulae we can re-express $T C_{2}(\pi)$ in terms of the pseudo-hermitian data:

$$
\widetilde{T} C_{2}(\pi)=\frac{i}{8 \pi^{2}}\left[\left(i / 2 R \theta^{\overline{1}} \wedge \theta^{1}+\bar{E} \theta^{1} \wedge \theta+E \theta^{\overline{1}} \wedge \theta\right) \wedge \theta_{1}^{1}-2 \theta \wedge \tau^{1} \wedge \tau^{\overline{1}}\right]
$$

Formula (3.6) defines $\widetilde{T} C_{2}(\pi)$ as a global form on $X$. To complete the construction we must show that, up to an exact form $\tilde{T} C_{2}(\pi)$ defines a unique form on $M$. Inserting (3.5) into (3.6) we obtain:

$$
\begin{aligned}
& i / 2 R \theta^{\overline{1}} \wedge \theta^{1}+\bar{E} \theta^{1} \wedge \theta+E \theta^{\overline{1}} \wedge \theta \\
& =-i / 2\left[R \theta^{1} \wedge \theta^{\overline{1}}+W \theta^{1} \wedge \theta-\bar{W} \theta^{\overline{1}} \wedge \theta\right] \\
& +1 / 6\left[\left(R_{1}-i W\right) \theta^{1}+\left(R_{\overline{1}}+i \bar{W}\right) \theta^{\overline{1}}\right] \wedge \theta .
\end{aligned}
$$

From Eqs. (2.9), (2.10) and (2.12) we see this equals:

$$
-2 i / 3 d \theta_{1}^{\mathbf{1}}+1 / 6 d(R \theta) \text {. }
$$

The local form can therefore be rewritten:

$$
\widetilde{T} C_{2}(\pi)=\frac{i}{8 \pi^{2}}\left[\left\{-2 i / 3 d \theta_{1}^{1}+1 / 6 d(R \theta)\right\} \wedge \theta_{1}^{1}-2 \theta \wedge \tau^{1} \wedge \tau^{\overline{1}}\right] .
$$

From (3.8) we easily see that $\tilde{T} C_{2}(\pi)$ defines a unique element in $H^{3}(M, \mathbf{R})$ : fix another coframe $\left\{\theta, \widetilde{\theta}^{1}, \widetilde{\theta}^{\mathrm{T}}\right\}$ with connection and torsion forms $\left\{\widetilde{\theta}_{1}^{1}, \tilde{\tau}^{1}, \tilde{\tau}^{\mathbb{1}}\right\}$. If $\theta^{1}=e^{i \alpha} \tilde{\theta}^{1}$, then

$$
\theta_{1}^{1}=\tilde{\theta}_{1}^{1}-i d \alpha, \text { and } \tau^{1}=e^{i \alpha} \tilde{\tau}^{1} .
$$

Thus, in terms of the new data we have: 


$$
\begin{aligned}
\widetilde{T} C_{2}(\pi)= & \frac{1}{8 \pi^{2}}\left[\left\{-2 i / 3 d \tilde{\theta}_{1}^{1}+1 / 6 d(R \theta)\right\} \wedge \tilde{\theta}_{1}^{1}-2 \theta \wedge \tilde{\tau}^{1} \wedge \tilde{\tau}^{\overline{1}}\right. \\
& \left.-i d\left\{\left(-2 i / 3 \tilde{\theta}_{1}^{1}+1 / 6 R \theta\right) \wedge d \alpha\right\}\right] .
\end{aligned}
$$

From these calculations and the triviality of the holomorphic tangent bundle of $M$ it follows that:

Theorem 3.1. There is a three-form $\widetilde{T} C_{2}(\pi)$ defined up to an exact form on a compact, 3-dimensional, strictly pseudoconvex $C R$ manifold $M$ with trivial holomorphic tangent bundle, such that $\left[T C_{2}(\pi)\right]=\pi_{Y}^{*}\left[\tilde{T} C_{2}(\pi)\right]$ in $H^{3}(Y, \mathbf{R})$. Here $[\cdot]$ denotes the deRham cohomology class of a closed form, and $\pi_{Y}$ is the projection from $Y$ to $M$.

Corollary 3.2. The evaluation of $\widetilde{T} C_{2}(\pi)$ on the fundamental class [M] in $H_{3}(M, \mathbf{R})$ is a biholomorphic invariant of the CR structure on $M$.

Remarks. 1) By integrating by parts we can obtain a local form defining $\left[\widetilde{T} C_{2}(\pi)\right]$ that depends on four derivatives of an immersion for a locally embedded $M$. The new form is:

$$
\widetilde{T} C_{2}(\pi)=\frac{i}{8 \pi^{2}}\left[-2 i / 3 d \theta_{1}^{1} \wedge \theta_{1}^{1}+1 / 6 R \theta \wedge d \theta_{1}^{1}-2 \theta \wedge \tau^{1} \wedge \tau^{\overline{1}}\right]+\text { exact }
$$

A careful check shows that the definition and normalization of $\theta_{1}^{1}$ requires three derivatives of an immersion, thereby substantiating our claim.

To simplify the notation, let us set

$$
\mu=\int_{M} \widetilde{T} C_{2}(\pi)
$$

2) If the holomorphic tangent bundle $T^{1,0} M$ is not trivial, but its $k$-th tensor power is trivial, then $X$ (or $Y$ ) admits a $k$-fold multi-section over $M$, and one may integrate $\widetilde{T} C_{2}(\pi)$ (or $T C_{2}(\pi)$ ) over the image of this multi-section. Dividing this by $k$ extends the definition of the invariant $\mu$ to $M$ with $c_{1}\left(T^{1,0} M\right)$ a torsion class. If $c_{1}\left(T^{1,0} M\right)$ is non-trivial in $H^{2}(M, \mathbf{R})$, then the class of $T C_{2}(\pi)$ is, up to a non-trivial constant factor, integral. To see this, note that if $c_{1}\left(T^{1,0} M\right)$ is non-trivial in $H^{2}(M, \mathbf{R})$, then the Gysin sequence of $X$ over $M$ shows that $H_{3}(X, \mathbf{R})$ is spanned by the inverse images in $X$ of two-cycles in $M$. On such a three-cycle, the two base-like forms in the formula above for $\widetilde{T} C_{2}(\pi)$ vanish identically. There remains the term involving $d \theta_{1}^{1} \wedge \theta_{1}^{1}$. Integrating out $\theta_{1}^{1}$ in the fiber direction, one is left integrating $d \theta_{1}^{1}$ over a two-cycle in $M$. But $d \theta_{1}^{1}$ represents $c_{1}\left(T^{1,0}\right)$ on $M$, substantiating the remark.

We next consider how the invariant $\mu$ behaves under deformation of the CR structure on $M$. We first calculate the first variation of $\mu$ with respect to a smooth 1-parameter family of CR structures on $M$, depending on $t \in(-\varepsilon, \varepsilon) \subset \mathbf{R}$. As a result of what has been proved above, we can calculate $\mu$ by calculating the matrix of one forms $\pi$ on $M$ in any frame $\left\{\theta, \theta^{1}, \theta^{\overline{1}}\right\}$ of $M$, and setting $\tilde{T} C_{2}(\pi)=\frac{1}{8 \pi^{2}} \operatorname{tr}(\pi \wedge \Pi+1 / 3 \pi \wedge \pi \wedge \pi)$. 
Proposition 3.3. $\delta \mu=-\frac{1}{4 \pi^{2}} \int_{M} \operatorname{tr}(\dot{\pi} \wedge \Pi)$.

Proof. Differentiating the definition of $\mu$, we get

$$
8 \pi^{2} \delta \mu=\int_{M} \operatorname{tr}(\dot{\pi} \wedge \Pi+\pi \wedge \dot{\Pi}+\dot{\pi} \wedge \pi \wedge \pi)
$$

Differentiating the definition of $\Pi$ with respect to $t$, one gets $d \dot{\pi}+\dot{\pi} \wedge \pi+\pi \wedge \dot{\pi}$ $=\dot{\Pi}$. Wedging with $\pi$ and taking traces, one concludes

$$
\operatorname{tr}(\pi \wedge \dot{\Pi})=\operatorname{tr}(\dot{\pi} \wedge \pi \wedge \pi+\pi \wedge d \dot{\pi}+\pi \wedge \dot{\pi} \wedge \pi) .
$$

Inserting (3.11) in (3.10) gives

$$
8 \pi^{2} \delta \mu=\int_{M} \operatorname{tr}(\dot{\pi} \wedge \Pi+\pi \wedge d \dot{\pi}+2 \pi \wedge \dot{\pi} \wedge \pi+\dot{\pi} \wedge \pi \wedge \pi)
$$

which by integration by parts and the symmetry of the trace

$$
\begin{aligned}
& =\int_{M} \operatorname{tr}(\dot{\pi} \wedge \Pi+d \pi \wedge \dot{\pi}+3 \pi \wedge \pi \wedge \dot{\pi}) \\
& =-2 \int_{M} \operatorname{tr}(\dot{\pi} \wedge \Pi),
\end{aligned}
$$

this last by the definition of $\Pi$ again, since $\operatorname{tr}(\Pi \wedge \pi) \equiv 0$.

Since there are no integrability conditions for a CR structure on a threedimensional manifold, it is quite easy to construct deformations of a given CR structure on $M$. We describe one method to do so, though point out that, by a theorem of J.W. Gray [G], this method gives the most general deformation of $\mathrm{CR}$ structure on $M$. We fix a smooth function $f=f(x, t)$ defined for $(x, t)$ in $M \times(-\varepsilon, \varepsilon) \subset M \times \mathbf{R}$ and such that $f(x, 0) \equiv 0$ on $M$. Fix a coframe $\left\{\theta, \theta^{1}, \theta^{\overline{1}}\right\}$ on $M$ such that $d \theta=i \theta^{1} \wedge \theta^{\overline{1}}$ on $M$. Define a new structure for $|t| \ll 1$ by declaring that the new structure has the same contact form $\theta$, while the new $\overline{T^{1,0} M}$ is defined by the vanishing of $\theta$ and $\theta^{1}+f \theta^{\mathrm{i}}$. If we define

$$
\theta_{f}^{1}=\left(1-|f|^{2}\right)^{-1 / 2}\left(\theta^{1}+f \theta^{\tilde{1}}\right),
$$

it is easy to check that

$$
d \theta=i \theta_{f}^{1} \wedge \theta_{f}^{\overline{1}} .
$$

For our purposes here, it suffices to consider $f$ of the form $f(x, t)=t f(x)$, where $f(x)$ is an arbitrary smooth function on $M$. Then, at $t=0$,

$$
\dot{\pi}=\left(\begin{array}{ccc}
* f \theta^{\overline{1}} & 0 \\
* & * & 2 i \bar{f} \theta^{1} \\
* & * & *
\end{array}\right) .
$$

Taking into account (3.4) above, we get for $\delta \mu$ at $t=0$ in this direction:

$$
-4 \pi^{2} \delta \mu=i \int\left(\bar{f} Q_{\overline{1}}^{1}+f Q_{1}^{\overline{1}}\right) \theta \wedge \theta^{1} \wedge \theta^{\overline{1}} .
$$


Since $f$ is arbitrary, we conclude immediately from this and the Bianchi identity:

Proposition 3.4. The invariant $\mu$ is stationary for a CR structure on $M$ if and only if the corresponding curvature $\Pi \equiv 0$ on $M$. In particular, if $M$ is simply connected, $\mu$ is stationary if and only if $M$ is CR-equivalent to the unit sphere in $\mathbf{C}^{2}$.

Remark. These propositions are analogues of Theorem 6.9 and Proposition 6.14 of [Ch-Si]. It is useful in understanding why several examples of computable $\mu$ in $\S 4$ below seem to give "topological" answers.

A calculation similar to that in Proposition 3.3 above gives the second variation $\delta^{2} \mu$ directly:

Proposition 3.5. $-4 \pi^{2} \delta^{2} \mu=\int_{M} \operatorname{tr}(\ddot{\pi} \wedge \Pi+\dot{\pi} \wedge d \dot{\pi}+2 \dot{\pi} \wedge \dot{\pi} \wedge \pi)$. The first term on the right drops out at critical points of $\mu$.

Remarks. 1) One will notice that for several examples where $\mu$ is calculated in $\S 4$ below, it appears to take on an absolute minimum, among the examples calculated, at $M$ with $\Pi \equiv 0$. We have checked that, at highest order terms, the second variation is non-negative at the standard structure on the unit sphere.

2) Consider the exotic contact structures of Lutz [L] on the three-sphere, and a non-degenerate CR structure on the sphere which has such an exotic structure as its underlying contact structure. This structure cannot be deformed to a $\mathrm{CR}$ structure with $\Pi \equiv 0$. To see this, one notes that such a deformation would be $\mathrm{CR}$ equivalent to the standard sphere, and the underlying contact structures would have the same homotopy invariant as the standard structure, a contradiction. This last is by Cerf's theorem that the group of orientation preserving diffeomorphisms of $S^{3}$ is connected. We do not know whether such exotic structures can be embedded as hypersurfaces in $\mathbf{C}^{2}$.

\section{§4. Examples}

In this section we will calculate the invariant $\mu$ for several families of CRmanifolds. The first set of examples are locally homogeneous. The form $\tilde{T} C_{2}(\pi)$ can thus be calculated simply in terms of a left invariant form on a threedimensional Lie group. There are two especially interesting examples here: the structures studied by Rossi in [Ro] on $S^{3}=S U(2)$, and a family of structures on a solvable group, some of which cover the boundary of a tube neighborhood of the torus $|z|=|w|=1$ in $\mathbf{C}^{2}$. For the first family, the value of $\mu$ is minimal exactly as the structure is embeddable in $\mathbf{C}^{2}$. The second family shows that $\mu$ is not, however, constant on all embedded perturbations of a given embedded hypersurface in $\mathbf{C}^{2}$. We note here that the hypersurfaces of this second class ara locally CR-equivalent, but are distinguished globally by the invariant $\mu$. Several examples considered here have $\Pi \equiv 0$ (i.e., are spherical in the sense of $[\mathrm{Bu}-\mathrm{Sh}]$ ). One of these families is recalculated below in a larger context.

The second set of examples are based on a calculation for the boundaries of Reinhardt domains in $\mathbf{C}^{2}$. This gives a second calculation for the tube neighbothoods of the torus $|z|=|w|=1$ as above, but also shows that Reinhardt 
perturbations of the standard structure on $S^{3} \subset C^{2}$ will vary the value of $\mu$. Thus, $\mu$ is not simply measuring something about the fundamental group of an embedded hypersurface (e.g., a holonomy invariant).

The final set of examples arise as the unit holomorphic tangent bundles for metrics of strictly positive or negative Gauss curvature on a compact Riemann surface. When the curvature of the given metric is constant, one recovers some of the spherical examples already encountered in the first set of examples above. Here the invariant $\mu$ takes an interesting form in terms of the curvature of the base Riemann surface. One also sees here again the tendency of $\mu$ to minimize at the spherical $M$ (case of constant Gauss curvature).

\section{Locally homogeneous examples}

$A$. We start with the examples on $S U(2)$, due to E. Cartan [C] and studied in the context of non-embeddability by Rossi [Ro]. Let $\alpha, \beta$, and $\gamma$ denote a basis of left-invariant one forms on $S U(2)$, with relations:

$$
d \alpha=-\beta \wedge \gamma, \quad d \beta=-\gamma \wedge \alpha, \quad d \gamma=-\alpha \wedge \beta .
$$

Setting $\theta=\gamma, \theta^{1}=1 / \sqrt{2}(\beta+i \alpha)$, we have:

$$
d \theta=i \theta^{1} \wedge \theta^{\overline{\mathrm{i}}}, \quad \mathrm{d} \theta^{1}=\mathrm{i} \theta \wedge \theta^{1} .
$$

This choice of $\theta, \theta^{1}$, describes the usual CR structure on $S^{3}$; in terms of the usual coordinates $(z, w)$ on $\mathbf{C}^{2}$, one can identify:

$$
\theta=-2 i(\bar{z} d z+\bar{w} d w), \quad \theta^{1}=\sqrt{2}(z d w-w d z) .
$$

The set of all $S U(2)$-invariant $\mathrm{CR}$ structures is obtained from the construction of $\S 2$ with $f \equiv 1$, i.e., with

$$
\theta^{1}=\left(1-t^{2}\right)^{-1 / 2}\left(\theta^{1}+t \theta^{\overline{1}}\right), \quad \mathrm{t} \in(-1,1)
$$

To simplify notation in what follows, set $c=c(t)=\left(\frac{1+t^{2}}{1-t^{2}}\right), s=s(t)=\frac{2 t}{1-t^{2}}$. The structure equations become

$$
\begin{gathered}
d \theta=i \theta_{t}^{1} \wedge \theta_{t}^{\overline{1}}, \\
d \theta_{t}^{1}=\theta_{t}^{1} \wedge(-i c \theta)+\theta \wedge(-i s) \theta_{t}^{\overline{1}} .
\end{gathered}
$$

Thus, $\theta_{1}^{1}=-i c \theta ; \tau^{1}=-i s \theta_{t}^{\overline{1}}$. We calculate that

$$
d \theta_{1}^{1}=c \theta_{t}^{1} \wedge \theta_{t}^{\overline{1}}
$$

and therefore $R=c$. The local form defining $\mu$ is therefore: 


$$
\begin{aligned}
\widetilde{T} C_{2}(\pi) & =\frac{1}{8 \pi^{2}} i\left(-c^{2} / 2+2 s^{2}\right) \theta \wedge \theta_{t}^{1} \wedge \theta_{t}^{\overline{1}} \\
& =-\frac{1}{16 \pi^{2}}\left(1-3 s^{2}\right) \theta \wedge d \theta=-\frac{1}{2 \pi^{2}}\left(1-3 s^{2}\right) d \text { Vol, }
\end{aligned}
$$

where $d$ Vol denotes the volume form of the unit sphere. Thus, $\mu=-\left(1-3 s^{2}\right)$.

$B$. Next we consider two spherical cases on three dimensional Lie groups. First we consider the nilpotent case, i.e., the three-dimensional Heisenberg group, $N$. Let $\alpha, \beta, \gamma$ be basis of left invariant forms with $d \alpha=d \beta=0, d \gamma=2 \alpha \wedge \beta$. Set $\theta=\gamma, \theta^{1}=\alpha+i \beta$. Then $d \theta=i \theta^{1} \wedge \theta^{\overline{1}}, d \theta^{1}=0$, and so $\theta_{1}^{1}=0, \tau^{1}=0$. Thus the structure is spherical, and the local form $\tilde{T} C_{2}(\pi) \equiv 0$. On the other hand, it is known that there are many discrete subgroups $\Gamma \subset N$ such that $M=\Gamma \backslash N$ is compact. For all such $M, \mu=0$. These $M$ can be interpreted geometrically as the unit circle bundle in a positive holomorphic line bundle over a compact Riemann surface of genus 1 , this bundle equipped with a special hermitian metric (cf. [Bu-Sh]).

We next consider a structure on $S L(2, \mathbf{R})$. We take a basis of the left invariant one froms $\alpha, \beta, \gamma$ such that $d \alpha=2 \gamma \wedge \beta, d \beta=-2 \gamma \wedge \alpha, d \gamma=2 \alpha \wedge \beta$. (This basis is dual to the basis $\left(\begin{array}{ll}0 & 1 \\ 1 & 0\end{array}\right),\left(\begin{array}{rr}1 & 0 \\ 0 & -1\end{array}\right)$, and $\left(\begin{array}{rr}0 & 1 \\ -1 & 0\end{array}\right)$ of $s \mathbb{I}(2, \mathbf{R})$.) Set $\theta=2 \gamma, \theta^{1}$ $=\sqrt{2}(\alpha+i \beta)$. Then $d \theta=i \theta^{1} \wedge \theta^{\overline{1}}$, and $d \theta^{1}=-i \theta \wedge \theta^{1}$. Comparing this with example 3 below, we see that this is the CR structure on the unit holomorphic tangent bundle of a Riemann surface of constant curvature -1 . We can also consider as above the deformations $\theta_{t}^{1}=\left(1-t^{2}\right)^{-1 / 2}\left(\theta^{1}+t \theta^{\overline{1}}\right)$. The structure equations are identical up to a sign to those in example 1 . A above. One finds

$$
\tilde{T} C_{2}(\pi)=-\frac{1}{16 \pi^{2}}\left(1-3 s^{2}\right) \theta \wedge d \theta .
$$

The evaluation of $\mu$ for $s=0$ is carried out in example 3 .

$C$. We next consider the case of solvable three-dimensional $G$. We limit ourselves to the following example admitting a cocompact discrete subgroup. We take the group $G$ to be the affine Euclidean group on $\mathbf{R}^{2}$ :

$$
\left\{\left(\begin{array}{ccc}
\cos \rho & \sin \rho & x \\
-\sin \rho & \cos \rho & y \\
0 & 0 & 1
\end{array}\right), \rho, x, y \in \mathbf{R}\right\} \text {. }
$$

We take a basis of left invariant one-forms $\alpha=d \rho, \beta=\cos \rho d x-\sin \rho d y, \gamma$ $=\sin \rho d x+\cos \rho d y$. Thus:

$$
d \alpha=0, \quad d \beta=-\alpha \wedge \gamma, \quad d \gamma=\alpha \wedge \beta .
$$


Set $\theta=2 \gamma, \theta^{1}=\alpha+i \beta$, so that $d \theta=i \theta^{1} \wedge \theta^{\overline{1}}$, and

$$
d \theta^{1}=\theta^{1} \wedge(-i / 4 \theta)+\theta \wedge\left(i / 4 \theta^{\overline{1}}\right),
$$

so that $\theta_{1}^{1}=-i / 4 \theta, \tau^{1}=i / 4 \theta^{\overline{1}}$. From this one calculates easily $\tilde{T} C_{2}(\pi)$ $=3 / 256 \pi^{2} i \theta \wedge \theta^{1} \wedge \theta^{\overline{1}}$. Let $L$ be a lattice subgroup of $G$, i.e., the set of all translations of $\mathbf{R}^{2}$ by elements in a lattice $L$ in $\mathbf{R}^{2}$, and let $M=L \backslash G$. Then for this $M, \mu=3 / 64 \pi$ Area $\left(L \backslash \mathbf{R}^{2}\right)$. For $L$ with orthogonal generators, $M$ can be embedded as a (Reinhardt) tube neighborhood of $|z|=|w|=1$ in $\mathbf{C}^{2}$. We thus see that there is no possible integrability condition on $\mu$ for embedded hypersurfaces.

Remark. The deformed structures defined by $\theta_{t}^{1}$ in 1.A and 1.B above are realized geometrically by two-sheeted covers of the intersection of the SO(3)- (resp., $S O(2,1)$-) invariant quadric in $\mathbf{C}^{3}$ with the level sets of the corresponding invariant hermitian form on $\mathbf{C}^{3}$. They can also be interpreted as the level sets of the metric form in the natural complex structure on the real tangent bundle of a rank one symmetric space.

\section{Reinhardt domains}

A Reinhardt domain $D$ in $\mathbf{C}^{2}$ can be described in terms of its generating curve in the $(\xi, \eta)$-plane, where, as usual, $\log |z|=\xi, \log |w|=\eta$. If $(\xi(s), \eta(s))$ is the generating curve, the boundary $M$ of $D$ is given as $\left\{\left(e^{\xi(s)+i \phi}, e^{\eta(s)+i \psi}\right)\right\}$. $M$ is strictly pseudoconvex if and only if the generating curve is strictly convex in $\mathbf{R}^{2}$ (in the standard Euclidean sense). These examples were suggested by Dave Barrett, see [Ba].

We normalize our parameter to be arc-length: $\dot{\xi}^{2}+\dot{\eta}^{2} \equiv 1$. Thus, $(\ddot{\xi}, \ddot{\eta})=$ $\kappa(-\dot{\eta}, \dot{\xi})$, where $\kappa$ is the curvature of the generating curve. On $M$, the contact form $\theta=\dot{\eta} d \phi-\dot{\xi} d \psi$, and we take $\theta^{1}=\sqrt{\kappa / 2}(i \dot{\xi} d \phi+i \dot{\eta} d \psi+d s)$, so that $d \theta$ $=i \theta^{1} \wedge \theta^{\overline{1}}$. One calculates that the connection form is

$$
\begin{aligned}
\theta_{1}^{1} & =i\left(\frac{\kappa_{s}}{\kappa}(\dot{\xi} d \phi+\dot{\eta} d \psi)-\frac{\kappa \theta}{2}\right) \\
& =\kappa_{s} / \sqrt{2 \kappa^{3}}\left(\theta^{1}-\theta^{\overline{1}}\right)-i / 2(\kappa \theta) .
\end{aligned}
$$

and the torsion form is $\tau^{1}=i \kappa / 2 \theta^{\overline{1}}$. The pseudo-hermitian curvature is obtained from

$$
d \theta_{1}^{1}=\left[\frac{\kappa}{2}-\frac{(\log \kappa)_{s s}}{\kappa}\right] \theta^{1} \wedge \theta^{\overline{1}}-\frac{3 i(\log \kappa)_{s}}{4 \sqrt{\kappa / 2}}\left[\theta^{1} \wedge \theta-\theta^{\overline{1}} \wedge \theta\right] .
$$

Therefore $R=\left[\kappa / 2-(\log \kappa)_{s s} / \kappa\right]$, and the local form is given by

$$
\tilde{T} C_{2}(\pi)=\frac{1}{8 \pi^{2}}\left[\frac{3 \kappa^{2}}{8}-\left[(\log \kappa)_{s}\right]^{2}+\frac{1}{6}(\log \kappa)_{s s}+\frac{1}{6}\left[\frac{(\log \kappa)_{s s}}{\kappa}\right]^{2}\right] \theta \wedge \theta^{1} \wedge \theta^{\overline{1}}
$$

Integrating out the angular variables we obtain: 


$$
\mu=\frac{1}{2} \int_{a}^{b} \kappa\left[\frac{3}{8} \kappa^{2}-\left[(\log \kappa)_{s}\right]^{2}+\frac{1}{6}(\log \kappa)_{s s}+\frac{1}{6}\left[\frac{(\log \kappa)_{s s}}{\kappa}\right]^{2}\right] d s,
$$

where $(a, b)$ is the interval of parametrization of the generating curve. If we consider the special cases $\kappa \equiv 1 / r$ then we obtain $\frac{3 \pi}{8 r^{2}}$. These are the same examples as in 1.C above. We remark once again that these examples show that there is no integrality condition on $\mu$ for embedded surfaces, and that since the generating curves for different values of $r$ are affinely equivalent by a homothety, the corresponding boundaries of Reinhardt domains in $\mathbf{C}^{2}$ are locally CR equivalent, and are distinguished globally by the invariant $\mu$.

We pursue this a bit further and consider the unbounded generating curve in $\mathbf{R}^{2}$ corresponding to the unit ball in $\mathbf{C}^{2}$, and consider Reinhardt perturbations of this domain. We will consider compactly supported variations of the curve $C=\left\{e^{2 \xi}+e^{2 \eta}=1\right\}$. Let $\xi=\xi(s), \eta=\eta(s)$ be the parametrization of $C$ by arc-length, and consider one-parameter families of curves $(\xi(s, t), \eta(s, t))$ with $\dot{\xi}^{2}+\dot{\eta}^{2} \equiv 1$, where $=$ the $s$-derivative. The first variation of such a curve is given by $(\delta \xi, \delta \eta)=\left.\frac{d}{d t}(\xi, \eta)\right|_{t=0}$, where

$$
\delta \eta=\int-(\dot{\xi} / \dot{\eta}) \frac{d}{d s}(\delta \xi) d s,
$$

and $\delta \xi$ is arbitrary in $\mathscr{C}_{0}^{\infty}$ save for the constraint

$$
\int_{-\infty}^{\infty} \frac{d}{d s}(\dot{\xi} / \dot{\eta}) \delta \xi d s=0
$$

It will be convenient to calculate in terms of $u:=\log \kappa$. In order for a function $v$ to be $\delta u$ for a variation of $C$ we must have

$$
v=\frac{\kappa}{\dot{\eta}} \frac{d^{2}}{d s^{2}}(\delta \xi)+\kappa^{2} \frac{\dot{\xi}}{\dot{\eta}^{2}} \frac{d}{d s}(\delta \xi)
$$

Since $\kappa$ and $\dot{\eta}$ are never 0 along $C$, we see that the set of such $v$ is of codimension 3 in $\mathscr{C}_{0}^{\infty}$. We calculate easily the second variation of $\mu$ with respect to such variations:

$$
\delta^{2} \mu=\int\left[\frac{1}{6}\left(\frac{d^{2}}{d s^{2}}(\delta u)\right)^{2}+A\left(\frac{d}{d s}(\delta u)\right)^{2}+B(\delta u)^{2}\right] \frac{1}{\kappa} d s,
$$

Where $A, B$ are real analytic functions of $s$. Thus $\delta^{2} \mu$ is essentially positive, and on any finite interval $[a, b]$ of $s$, we may thus find an infinite dimensional family of admissible $\delta \xi$, and corresponding $\delta u$ such that $\delta^{2} \mu>0$ in that direction. Thus $\mu$ is not constant on smooth perturbations of the sphere in $\mathbf{C}^{2}$. In particular, $\mu$ is not a holonomy-type invariant for embedded hypersurfaces. (Equation (4.6) below, suitably interpreted, gives a more precise statement on the positivity of $\delta^{2} \mu$.) 


\section{Tangent circle bundles over Riemann surfaces}

In this last set of examples, we consider a compact Riemann surface $\Sigma$ with a hermitian metric $h$. Let $M$ be the unit circle bundle in $T^{1,0} \Sigma$, the holomorphic tangent bundle to $\Sigma . M$ is a $U(1)$ bundle over $\Sigma$, and on it there is a canonical C-valued one-form $\Theta^{1}$, the dual coframe. Since $\operatorname{dim} \Sigma=2, h$ is a Kähler metric, and there is therefore a unique torsion free connection form $\Theta_{1}^{1}$ so that

$$
d \Theta^{1}=\Theta^{1} \wedge \Theta_{1}^{1} .
$$

The form $\Theta_{1}^{1}$ is $i \mathbf{R}$-valued, and $U(1)$-invariant on $M$. The Gauss equation implies that

$$
d \Theta_{1}^{1}=K \Theta^{1} \wedge \Theta^{\overline{1}}
$$

where $K$ is the Gauss curvature. If we begin with (4.2) it says that $-i \Theta_{1}^{1}$ and $\Theta^{1}$ define a CR structure on $M$; it will be non-degenerate if $K$ never vanishes. With this assumption on $K$, set

so that:

$$
\theta=i \operatorname{sign}(K) \Theta_{1}^{1}, \quad \theta^{1}=\sqrt{|K|} \Theta^{1}
$$

$$
d \theta=i \theta^{1} \wedge \theta^{\overline{1}} .
$$

To compute the pseudo-hermitian connection, we use (4.1) and (4.3) to obtain:

$$
d \theta^{1}=\theta^{1} \wedge\left[\frac{1}{2}(\log |K|)_{1} \theta^{1}-\frac{1}{2}(\log |K|)_{\overline{1}} \theta^{\overline{1}}-i \operatorname{sign}(K) \theta\right] .
$$

From (4.5) it follows that the torsion is 0 . We consider first the special case of constant $K$. Then the pseudo-hermitian curvature $R$ is $\operatorname{sign}(K)$, and for the local form we get:

$$
\widetilde{T} C_{2}(\pi)=\frac{1}{8 \pi^{2}}\left[\frac{-1}{2}\right] K \Theta_{1}^{\mathrm{l}} \wedge \Theta^{1} \wedge \Theta^{\overline{1}}
$$

Integrating over the fiber gives $\operatorname{sign}(K) \cdot 2 \pi i$ and thus the value of the characteristic number is:

$$
\frac{-1}{8 \pi} \int_{\Sigma}|K| d \text { Area }=\frac{-|\chi(\Sigma)|}{4}
$$

here $\chi(\Sigma)$ is the Euler characteristic of $\Sigma$. This agrees with our earlier calculations above in 1.A and 1.B: in particular, $\mathbf{R} \mathbf{P}^{3}$ is the unit tangent bundle for the standard Riemannian structure on $S^{2}$ and $S^{3}$ is a double cover, so $\mu$ should be $-1 / 2$. Note that the value of $\mu$ is independent of the conformal structure on $\Sigma$, consistent with 3.4 and example 1 .B above.

To compute the general case we need to compute $d \theta_{1}^{1}$ from (4.4). We obtain:

$$
\begin{aligned}
d \theta_{1}^{1}= & d\left((\log |K|)_{1}\right) \wedge \frac{\theta^{1}}{2}-d\left((\log |K|)_{\overline{1}}\right) \wedge \frac{\theta^{\overline{1}}}{2} \\
& +(\log |K|)_{1} \frac{d \theta^{1}}{2}-(\log |K|)_{\overline{1}} \frac{d \theta^{\overline{1}}}{2}-i \operatorname{sign}(K) d \theta .
\end{aligned}
$$


As $\log |K|$ is a function on $\Sigma$,

$$
d\left((\log |K|)_{1}\right) \wedge \theta^{1}=(\log |K|)_{1 \overline{1}} \theta^{\overline{1}} \wedge \theta^{1} .
$$

On the other hand, $(\log |K|)_{1} d \theta^{1}=(\log |K|)_{1} \theta^{1} \wedge \theta_{1}^{1}$, and so:

$$
\begin{aligned}
d \theta_{1}^{1} & =\left(K-\left(\log \mid K D_{1 \overline{1}}\right) \Theta^{1} \wedge \Theta^{\overline{1}}\right. \\
& =\left(\operatorname{sign}(K)-\frac{\left(\log |K|_{1 \overline{1}}\right.}{|K|}\right) \theta^{1} \wedge \theta^{\overline{1}} .
\end{aligned}
$$

Thus, the pseudo-hermitian curvature is

$$
R=\left(\operatorname{sign}(K)-\frac{(\log |K|)_{1} \bar{i}}{|K|}\right)
$$

The local form becomes:

$$
\tilde{T} C_{2}(\pi)=\frac{i}{8 \pi^{2}}\left[\frac{-2}{3}\left(1-\frac{(\log |K|)_{1} \overline{1}}{K}\right)+\frac{1}{6}\left(\operatorname{sign}(K)-\frac{(\log |K|)_{1} \overline{1}}{|K|}\right)^{2}\right] \times \theta \wedge \theta^{1} \wedge \theta^{\overline{1}} .
$$

Simplifying,

$$
\begin{aligned}
\tilde{T} C_{2}(\pi) & =\frac{i}{8 \pi^{2}}\left[-\frac{1}{2}+\frac{1}{3} \frac{(\log |K|)_{1 \overline{1}}}{K}+\frac{1}{6}\left(\frac{(\log |K|)_{1 \overline{1}}}{K}\right)^{2}\right] \theta \wedge \theta^{1} \wedge \theta^{\overline{1}} \\
& =\frac{i}{8 \pi^{2}}\left[-\frac{1}{2}+\frac{1}{3} \frac{(\log |K|)_{1 \overline{1}}}{K}+\frac{1}{6}\left(\frac{(\log |K|)_{1 \bar{i}}}{K}\right)^{2}\right] i K \Theta_{1}^{1} \wedge \Theta^{1} \wedge \Theta^{\overline{1}} .
\end{aligned}
$$

Integrating over $M$, we get

$$
\mu=\frac{-|\chi(\Sigma)|}{4}+\frac{1}{24 \pi} \int_{\Sigma}[\Delta(\log |K|)]^{2} \frac{d \text { Area }}{|K|}
$$

For a surface of fixed genus we see that the minimum of this functional is attained only at the constant curvature metrics.

\section{References}

[Ba] Barrett, D.: A remark on the global embedding problem for three-dimensional CR-manifolds. Preprint (1986)

[Bu-Sh] Burns, D., Shnider, S.: Spherical hypersurfaces in complex manifolds. Invent. Math. 33, 223-246 (1976)

[C] Cartan, E.: Sur la géometrie pseudo-conforme des hypersurfaces de l'espace de deux variables complexes, I. Ann. Mat. 11, 17-90 (1932); II. Ann. Sc. Ec. Norm. Super. Pisa. Cl. Sci., IV. Ser. 1, 333-354 (1932)

[( h-Mo] Chern, S.S., Moser, J.: Real hypersurfaces in complex manifolds. Acta Math. 133, 219-271 (1974)

[( $\mathrm{B}-\mathrm{Si}]$ Chern, S.S., Simons, J.: Characteristic forms and geometric invariants. Ann. Math. 99, 48-69 (1974)

[Lo] Donelly, H.: Eta invariants and the boundaries of Hermitian manifolds. Am. J. Math. 99, 879-900 (1976) 
[G] Gray, J.W.: Some global properties of contact structures. Ann. Math. 69, 421-450 (1959)

[Ko-Oc] Kobayashi, S., Ochiai, T.: $G$-structures of order two and transgression operators. J. Differ. Geom. 6, 213-230 (1971)

[L] Lutz, R.: Structures de contact sur les fibrés principaux en cercles de dimension trois. Ann. Inst. Fourier 27, (3) 1-15 (1977)

[Ro] Rossi, H.: Attaching analytic spaces to an analytic space along a pseudoconcave boundary. In: Aeppli, A., Calabi, E., Röhrl, R. (eds.) Proceedings of the Conference on complex manifolds. Berlin-Heidelberg-New York: Springer 1965

[We] Webster, S.: Pseudo-hermitian structures on a real hypersurface. J. Differ. Geom. 13, 25-41 (1978)

[Y] Yau, S.S.-T.: Kohn-Rossi cohomology and its application to the complex Plateau problem, I. Ann. Math. 113, 67-110 (1981)

Oblatum 6-III-1987 \& 29-X-1987 